\title{
Concurrent Design in Software Development Based on Axiomatic Design
}

\section{Ruihong Zhang, Jianzhong Cha, Yiping Lu}

Beijing Jiaotong University, Beijing, PR China

Email: ruihong0613@yahoo.com.cn

CE2007, San Jose dos Campos, Brazil, July 16th to 20th 
$>$ Introduction

$>$ Concurrent Design in Software Development Based on Axiomatic Design

$>$ Case Study: AD Based Software Development

$>$ Conclusions 


\section{Introduction}

\section{Software development \\ system engineering}
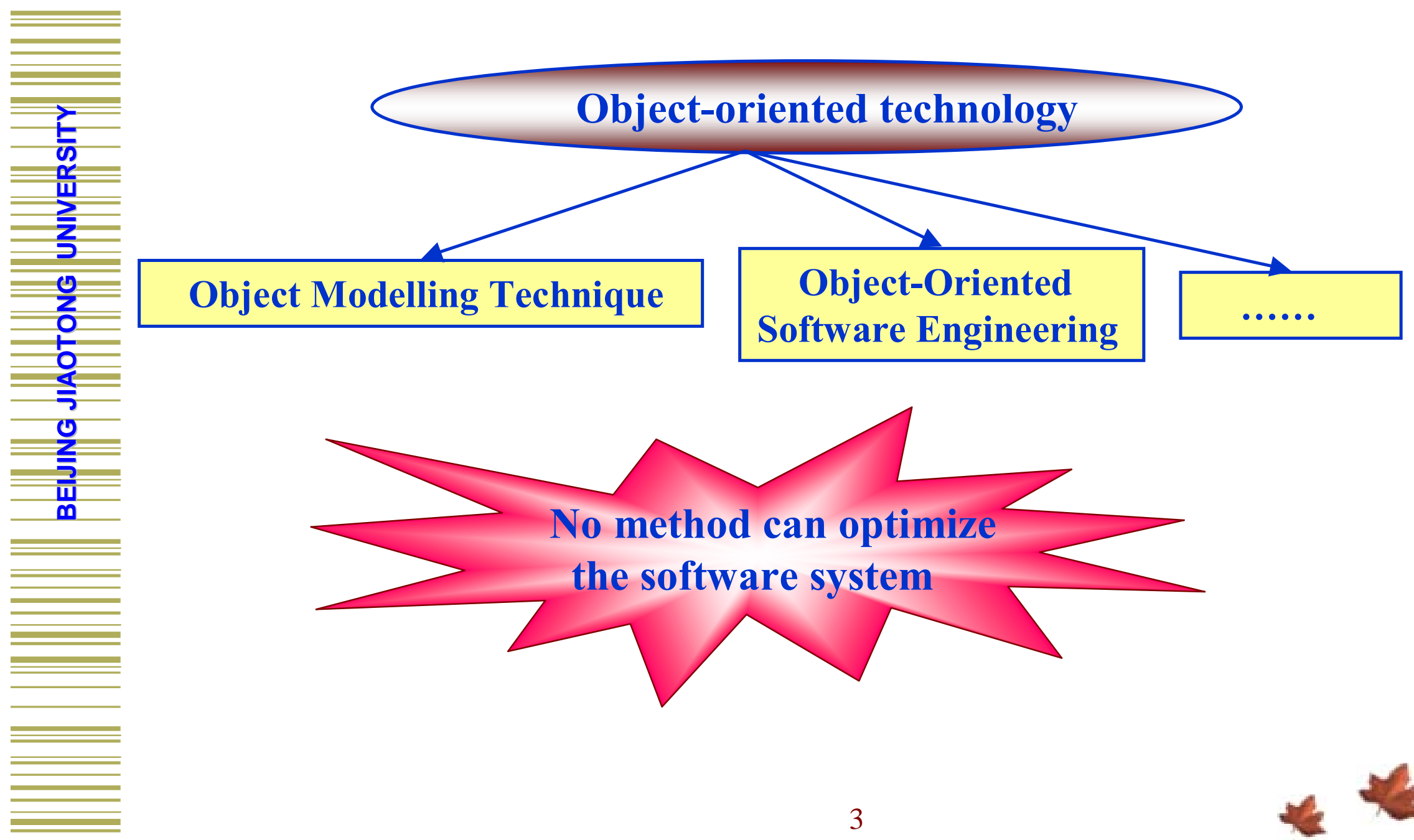


\section{Cont'd}

\section{Concurrent design}
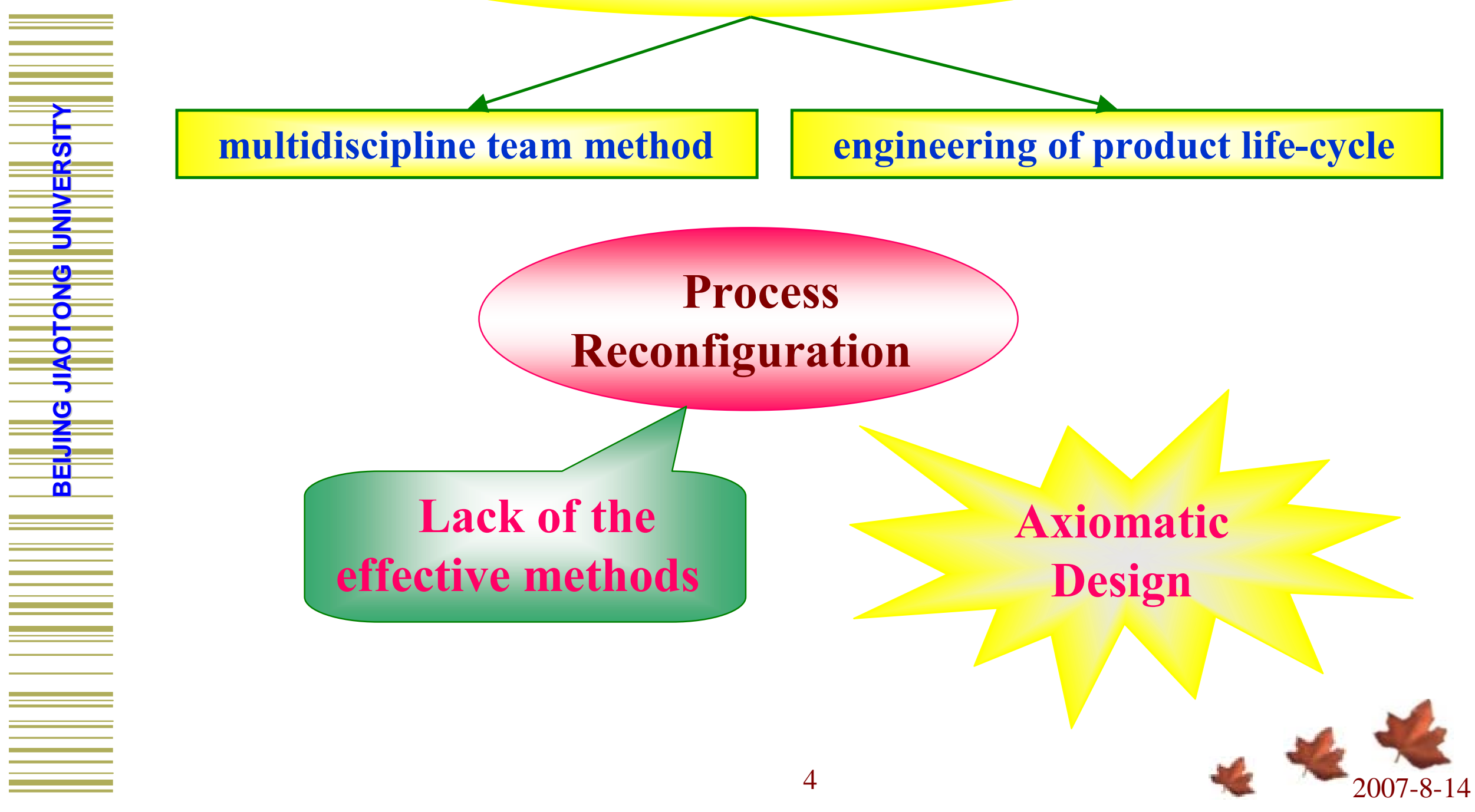


\section{Concurrent Design in Software}

Development Based on Axiomatic Design

\subsection{Background of Axiomatic Design}
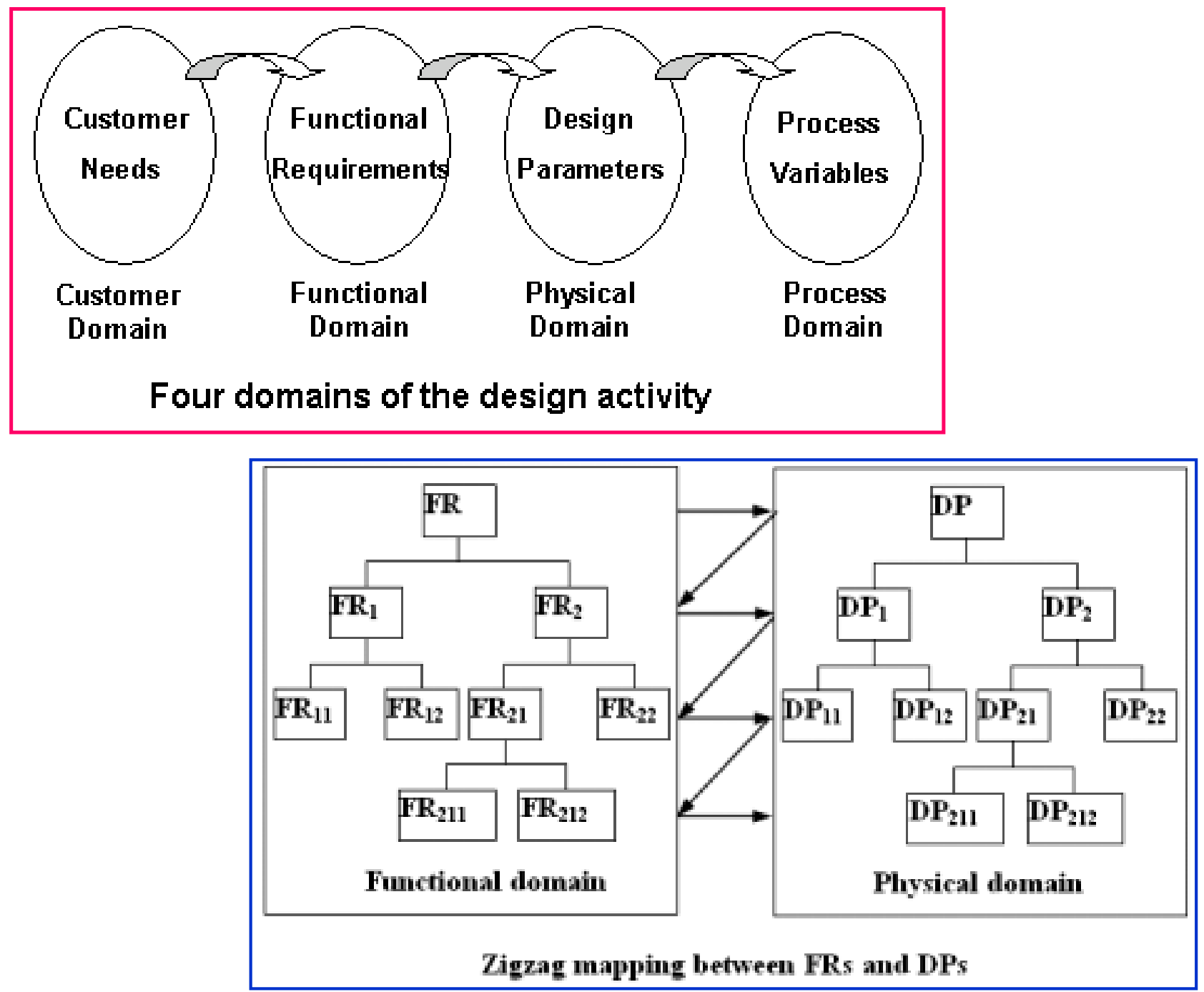


\section{Design equation for product design:}

\section{$\{F R \mathrm{~s}\}=[A]\{D P \mathrm{~s}\}$ \\ Design matrix}

$[A]=\left[\begin{array}{ccc}A_{11} & A_{12} & A_{13} \\ A_{21} & A_{22} & A_{23} \\ A_{31} & A_{32} & A_{33}\end{array}\right] \begin{aligned} & \text { Diagonal matrix } \rightarrow \text { Uncoupled design } \sqrt{ } \\ & \text { Triangular matrix } \rightarrow \text { Decoupled design } \sqrt{ } \\ & \text { Other } \rightarrow \text { Coupled design }\end{aligned}$ 
- Uncoupled design -- design tasks are independent by nature and can be concurrently processed

- Decoupled design -- design tasks can be decoupled into triangle matrix, which should be processed by sequence

- Coupled design - design tasks are coupled so iterative design process is necessary 


\section{Cont'd}

\section{- AD method vs Object-oriented technology}

\begin{tabular}{|l|}
\hline Object $=\mathrm{FR}$ \\
\hline Attribute \\
Data structure $=\mathrm{DP}$ \\
\hline Method \\
$\mathrm{FR}_{i}=\mathrm{A}_{i j} \mathrm{DP}_{j}$ \\
\hline
\end{tabular}

Graphic representation of an object

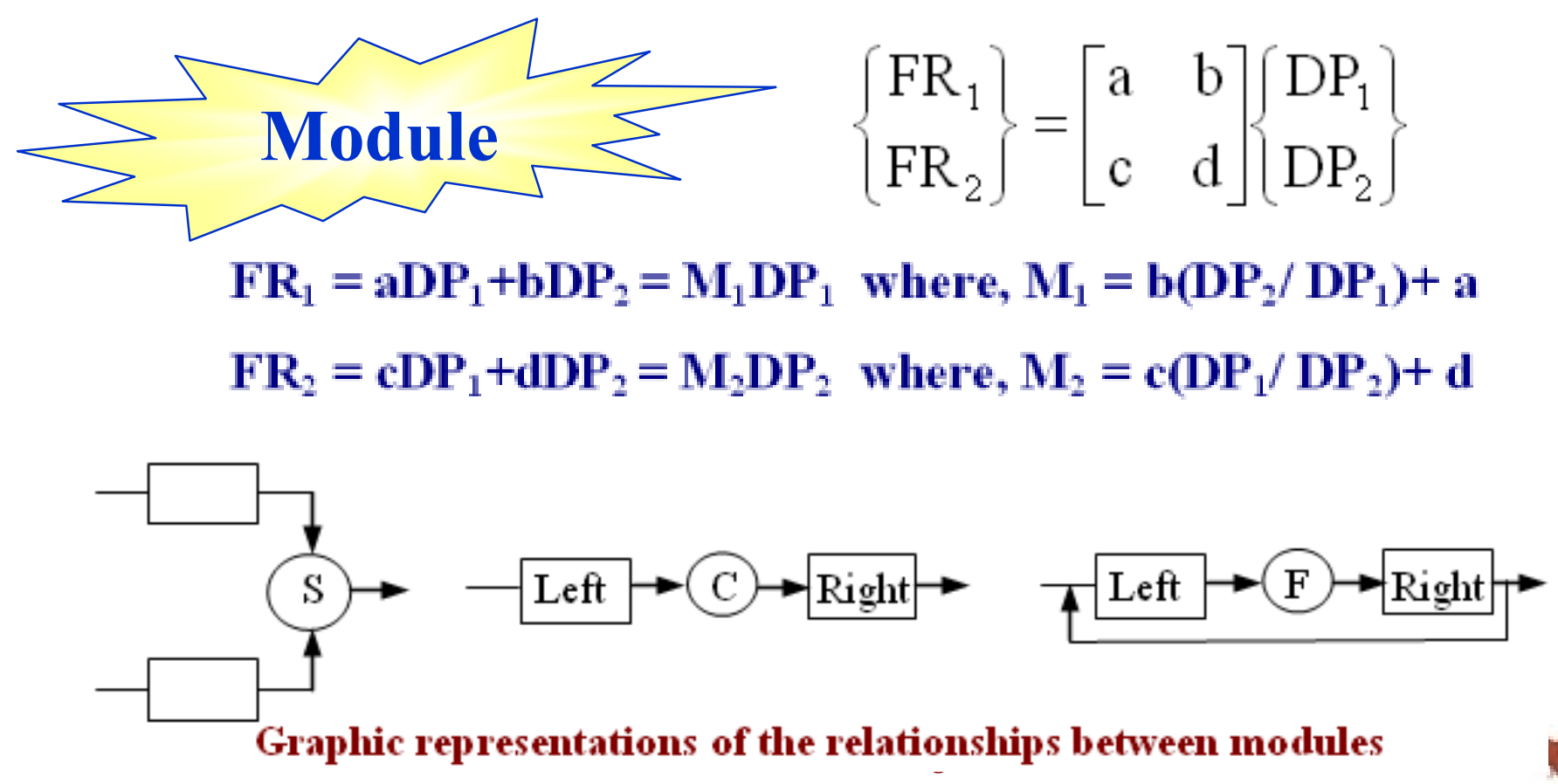

$\left\{\begin{array}{l}\mathrm{FR}_{1} \\ \mathrm{FR}_{2}\end{array}\right\}=\left[\begin{array}{ll}\mathrm{a} & \mathrm{b} \\ \mathrm{c} & \mathrm{d}\end{array}\right]\left\{\begin{array}{c}\mathrm{DP}_{1} \\ \mathrm{DP}_{2}\end{array}\right\}$

$\mathrm{FR}_{1}=\mathrm{aDP}_{1}+\mathrm{bDP}_{2}=\mathrm{M}_{1} \mathrm{DP}_{1}$ where, $\mathrm{M}_{1}=\mathbf{b}\left(\mathrm{DP}_{2} / \mathrm{DP}_{1}\right)+\mathbf{a}$

$\mathrm{FR}_{2}=\mathrm{cDP}_{1}+\mathrm{dDP}_{2}=\mathrm{M}_{2} \mathrm{DP}_{2}$ where, $\mathrm{M}_{2}=\mathbf{c}\left(\mathrm{DP}_{1} / \mathrm{DP}_{2}\right)+\mathrm{d}$ 

Development Based on AD Method

- Step 1: Analyzing the software with AD method

- Step 2: Defining modules of the software

- Step 3: Reconfiguring the sequence of modules 


\section{Case Study - AD Based Software Development}

\subsection{Analyzing and Designing}

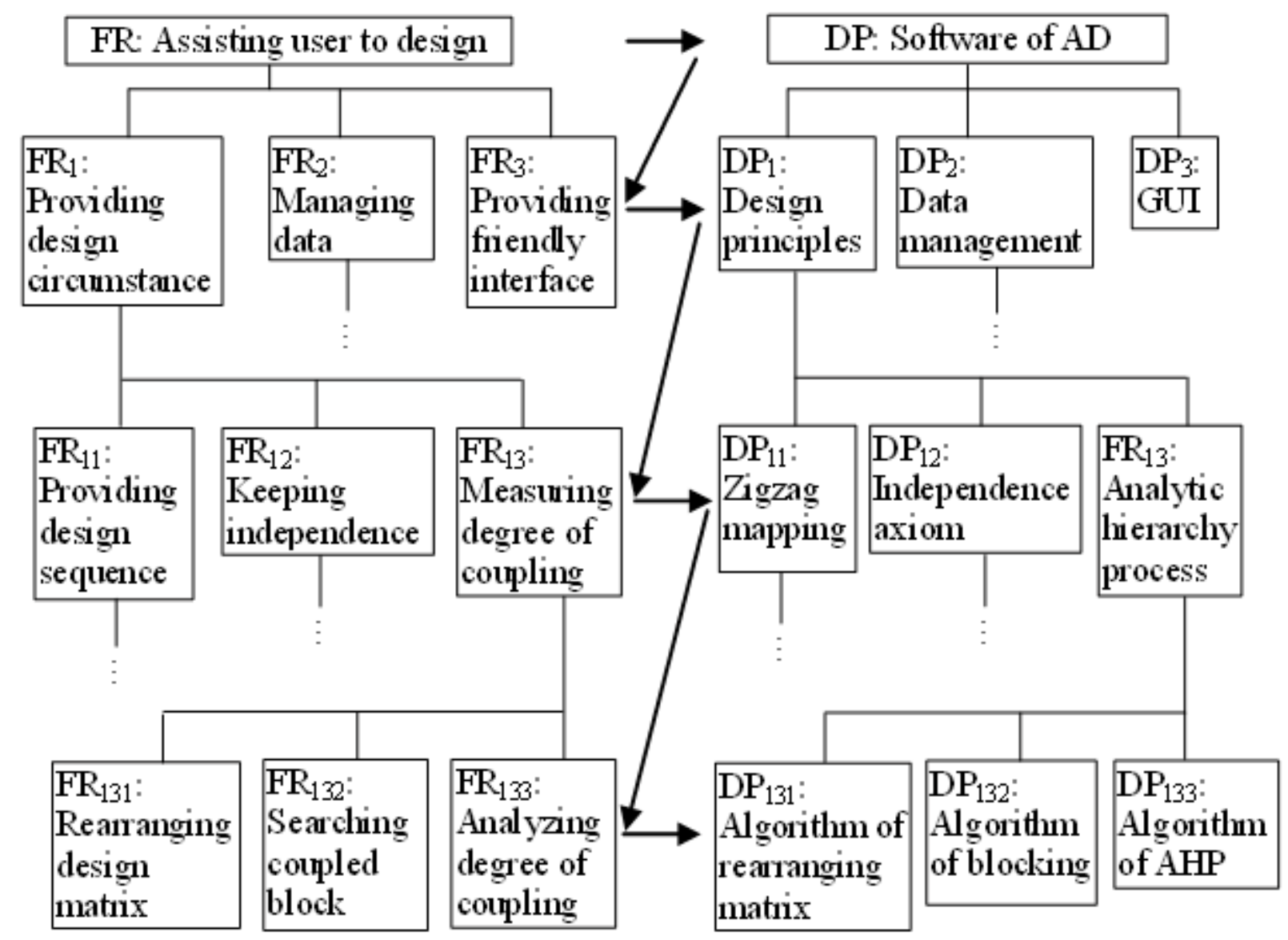

Functional-structure model 
Full design matrix

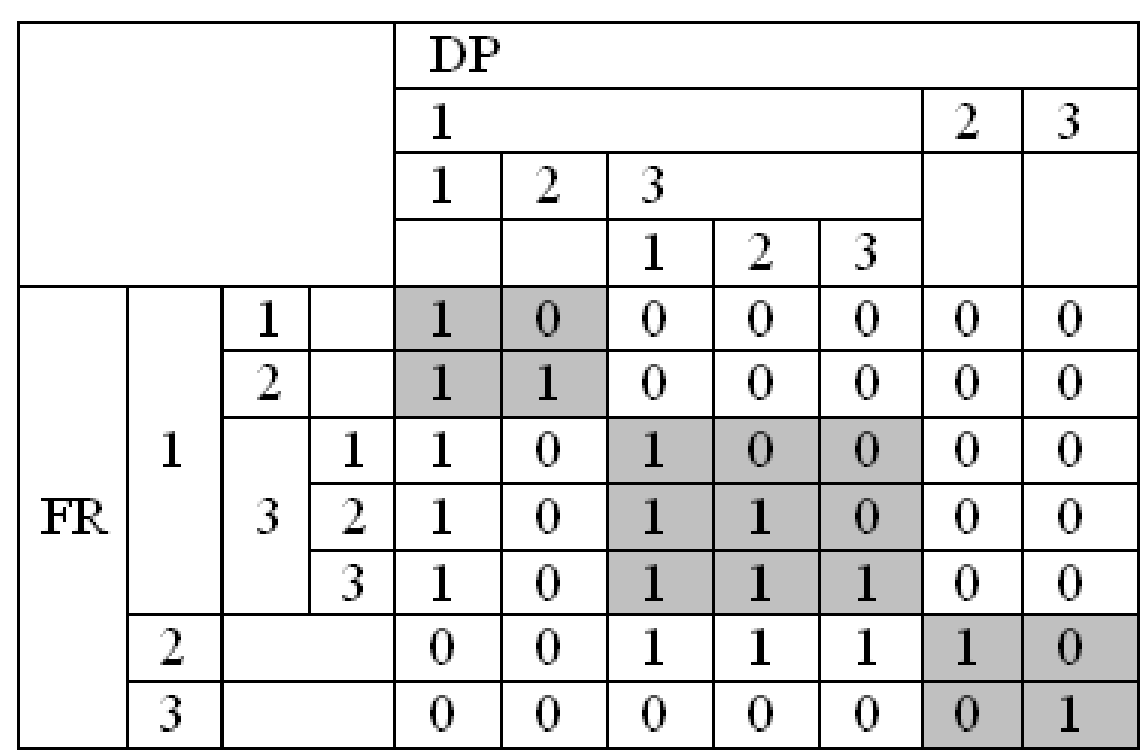


- Zigzag mapping

- Independence axiom

- Algorithm of rearranging matrix

- Algorithm of blocking

- Algorithm of AHP

- Data management

- GUI (Graphical User Interface) 


\subsection{Reconfiguring the Sequence of Modules}

Design tasks are decoupled, so that these modules must be performed in sequence.

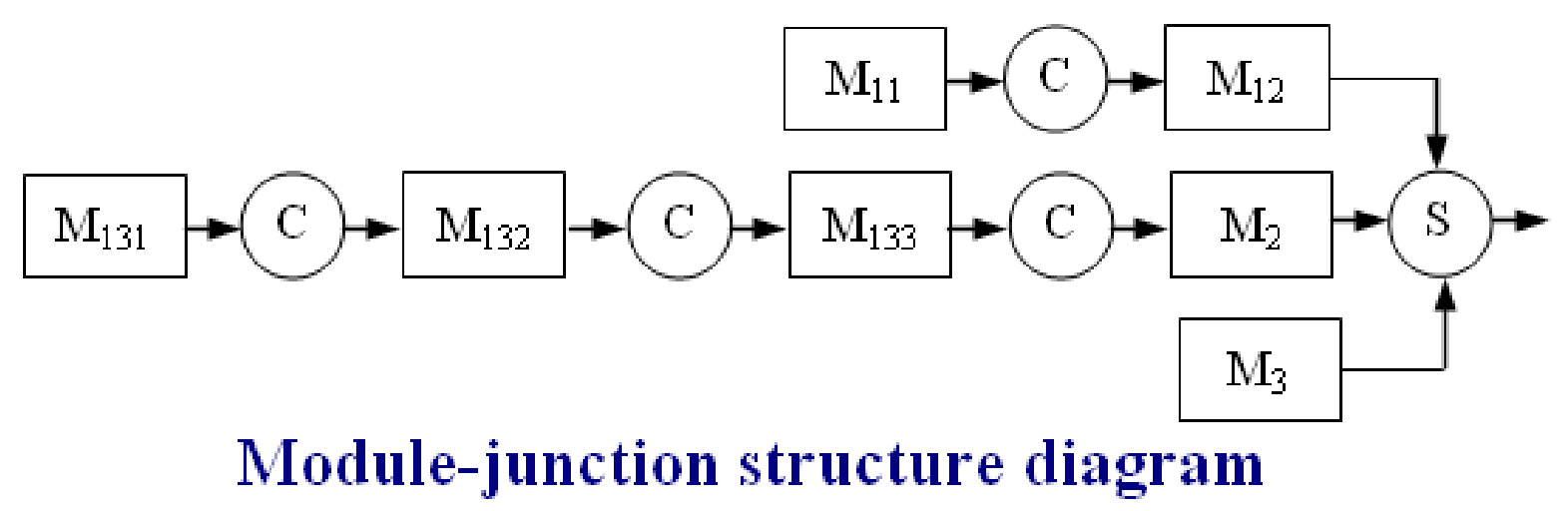

$>$ "S" indicates modules can be concurrently implemented.

$>$ " $C$ " indicates modules should be implemented sequentially with arrow direction 


\section{Conclusions}

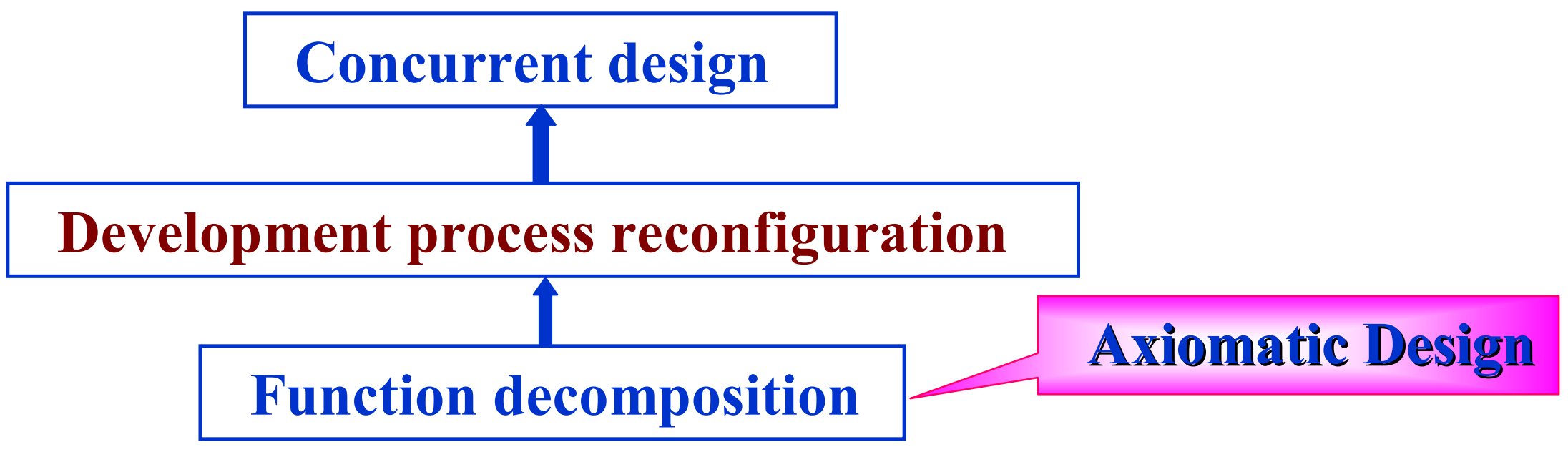

- Software design tasks can be categorised by uncoupled design and decoupled design with AD method

- Uncoupled design tasks can be concurrently carried out with significantly shorter overall developing time

Decoupled design modules should be processed in sequence so that the development process can be managed effectively

$\checkmark$ Relationship between software modules is established by analyzing the design matrix with AD method 


\title{
THANKS FOR YOUR ATTENTION!
}

\author{
Q\&A
}

